\title{
To Reduce The Handover Delay In Wimax When The Mobile Station Moves At Higher Speed By Using The Save Point.
}

\author{
Mrs Sukhpreet Kaur, Jasbir Kaur
}

\begin{abstract}
The IEEE 802.16e standard (i.e., mobile WiMAX) has been proposed to provide the connectivity in wireless networksfor mobile users (including users at a vehicular speed). It is shownin our analysis that the probability of a successful handoverdecreases significantly when the user moves at a higher speed. In this paper we have compared three techniques Forward Error Correction (FEC) with retransmission to offer extra protection for handover signaling messages to enhance the probability of a successful handover, especially at a higher velocity, without using a save point between source and destination and using a save point between the source and destination. Comparison between their line graphs is also given in this paper.
\end{abstract}

\section{Introduction}

The rapid growth of high data rate services in wireless communication networks demands new technologies for broadband wireless access. The Worldwide Interoperability for Microwave Access (WiMAX) is an emerging technology that enables the delivery of the last mile wireless broadband access as an alternative to the wired broadband access such as cable and DSL. It provides a convenient way to build a wireless metropolitan area network (WMN), which offers a broad range of high data rate applications, such as broadband Internet access, Voice over Internet Protocol (VoIP), Internet Protocol Television (IPTV), etc. to wireless users anytime and anywhere. For more technical details, we refer to the newly developed IEEE 802.16 standard [1], [2]. Handover is one of essential issues in mobile wireless communications since it is needed to maintain uninterrupted services during user's movement from one location to another [3], [4]. It manages mobility between subnets in the same network domain (micro-mobility) and between two different network domains. The current mobile WiMAX standard defines handover operations to support micro-mobility for the point-tomultipoint (PMP) mode communication. That is, it handles the mobile subscriber station (MS) switching from one base station to another. In general, handover techniques can be divided into soft handover (SHO) and hard handover

(HHO). SHO employs a make-before-break approach where aconnection to the next base station (BS) is established before an MS releases an ongoing connection to the original BS. It guarantees zero break-time during handover at the cost This work is supported in part by the China Scholarship Council (CSC). of lower spectral efficiency. This technique is suitable for latency-sensitive services such as voice communications, video conferencing, and multi-player gaming. In contrast, HHO uses a break-before-make approach where a connection with the serving BS is terminated before a mobile station switches to another BS. HHO is more bandwidth-efficient than SHO. However, it has a nonzero breaktime, thus leading to longer delay in service delivery. A typical mobile WiMAX network uses packet-switching with mostly bursty delay-tolerant data traffic. Therefore, HHO is also used in mobile WiMAX. However, for certain delay-sensitive applications, HHO must be optimized to meet the required quality of service (QoS). Recently, a large amount of research has been conducted to address the QoS of HHO in mobile WiMAX. They focused their attention on the choice of the best target BS through tracking the MS movement, analyzing the QoS information of neighbor BSs or detecting the signal level from BSs [6], [7]. In [8], data packet loss during handover was reduced via caching in the upper layer devices such as routers. To reducedelay during the handover process, Chen et al. [9] proposed aprecoordination mechanism, which can reduce the handoverlatency by measuring the distance between the BS and the MS,predicting the time that handover occurs, and pre-allocatingavailable resource for handover usage.

Besides, it was shownin [9] that the handover delay could be further reduced throughtransmitting MAC layer messages via the IP layer. Jiao et al.[11] proposed a connection identification (CID) assignmentstrategy to avoid conflicts of the CID assignment during thehandover procedure. Hu et al. [12] applied a different handovermechanism to classified data. Both of them can decrease datadelay in handover.Most previous work considered the handover performancefrom the viewpoint of the MAC or IP layer, but paid littleattention to the physical layer. That is, physical channelquality and/or user's mobility are not taken into account.Usually, handover occurs in the boundary of BS's coveragearea, where signals transmitted on the radio channel are weakand unstable. When channel quality degrades, both signaling messages and data packets are at a higher risk of being lostor corrupted. Moreover, user's mobility plays an importantrole in the handover process. The radio channel quality formobile users at a higher velocity often suffers from severe degradation due to the Dopploar frequency shift. Since the overlap area is limited between adjacent BSs, the requirement on handover latency is 
more stringent for users with higher mobility. In order to support full mobility (which means seamingless handover for users moving at a speed of 120 kilometer per hour or higher), these issues become severer and have to be addressed. In this paper we use three techniques FEC with retransmission, without save point and with save point.

\section{Fec With Retransmission}

To increase the successful handover probability of an MS at a vehicular speed, we need to establish more reliable wireless links in transmitting signaling messages besides using auto retransmission scheme. In this section, we investigate the improvement of the probability of a successful handover by adding extra protection for handover signaling messages. Forward error correction codes provide an error control technique for data transmission, where a sender adds redundant data to messages. These redundant data can assist the receiver detecting and correcting errors to further improve wireless channel quality. During the WiMAX handover procedure, signaling messages mostly consist of small data packets whose size is less than 50 bytes.

Consequently, the amount of parity-check data needed for error correction in each message is small and the resource required by error correction does not increase excessively. In comparison, the cost of the only retransmission scheme is relatively higher. In other words, this method offersan efficient solution to improve the handover performance. Different FEC schemes have different error correcting capabilities. For the same type of FEC codes, the more the redundant data, the higher the error correction capability. For a given MS velocity, we can estimate the size of FEC codes in order to achieve the desired successful handover probability. And for the sake of simple, we can use the average successful message probability the higher the velocity of an MS, the more the number of bits needed in an adaptive FEC scheme to achieve the target successful handover probability. For example, to achieve a probability of $80 \%, 2,10$ and 28 bits have to be added into each handover signaling message when the MS is moving at a speed of 50,70 and $90 \mathrm{~km} / \mathrm{h}$, respectively. Then, to achieve the target probability, we can adopt an adaptive FEC scheme that uses a different redundant bit size according to the MS velocity. The line graph of FEC with retransmission is give below.

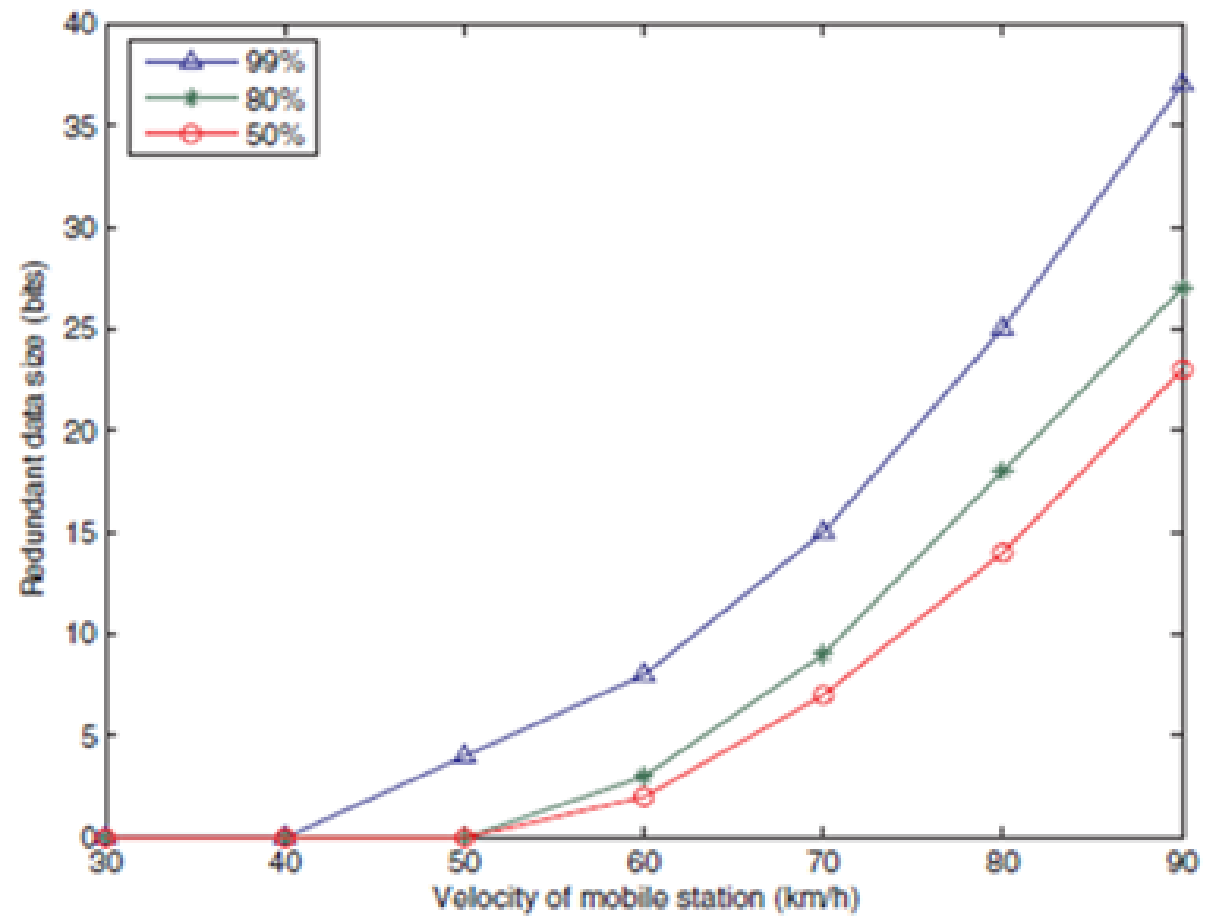

This method is only efficient for light loaded networks but for the heavy loaded networks this technique will not work.

III. Without Using The Save Point Between The Source And Destination

When we use no save point between the source and the destination, when the user moves at higher speed ie more than 900 packets per second then the data loss started due to which the hand over delay occurs. The diagram of the without save point is given below 

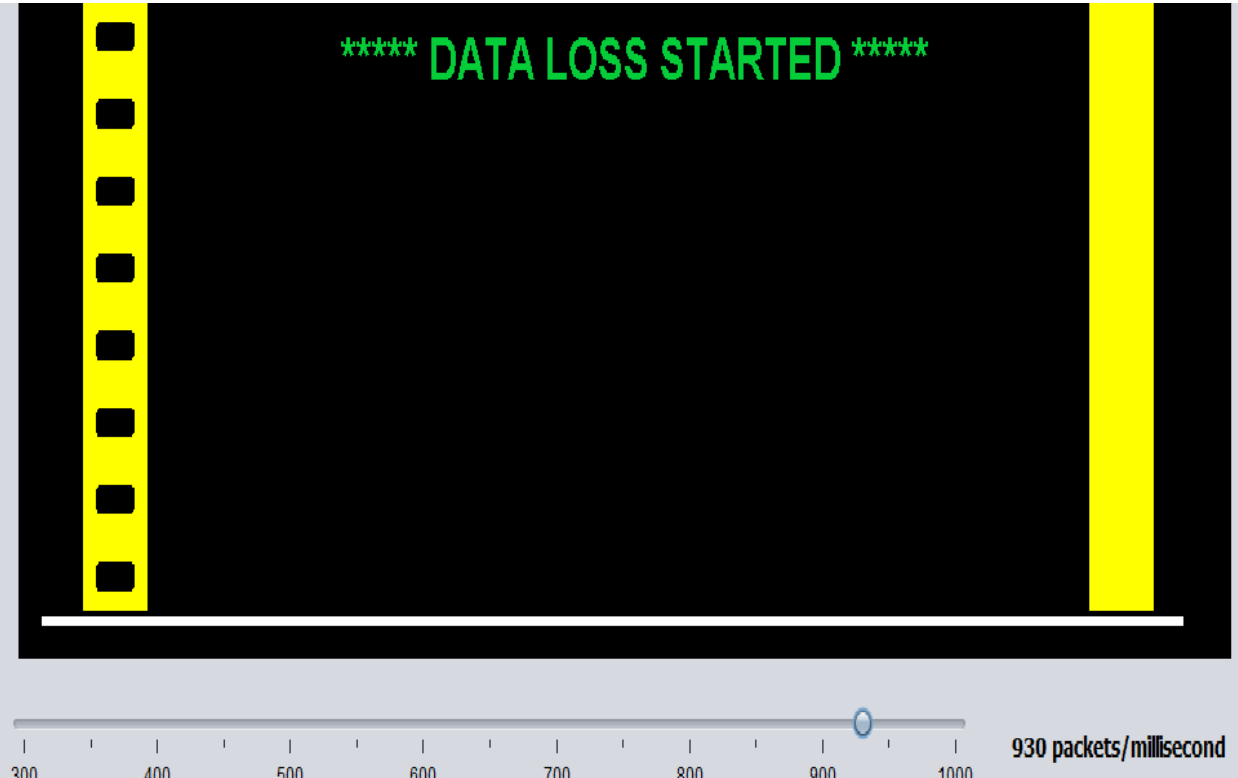

as given in the figure the data loss started at the speed of 930 packets/millisecond. The line graph of the above consideration is given below

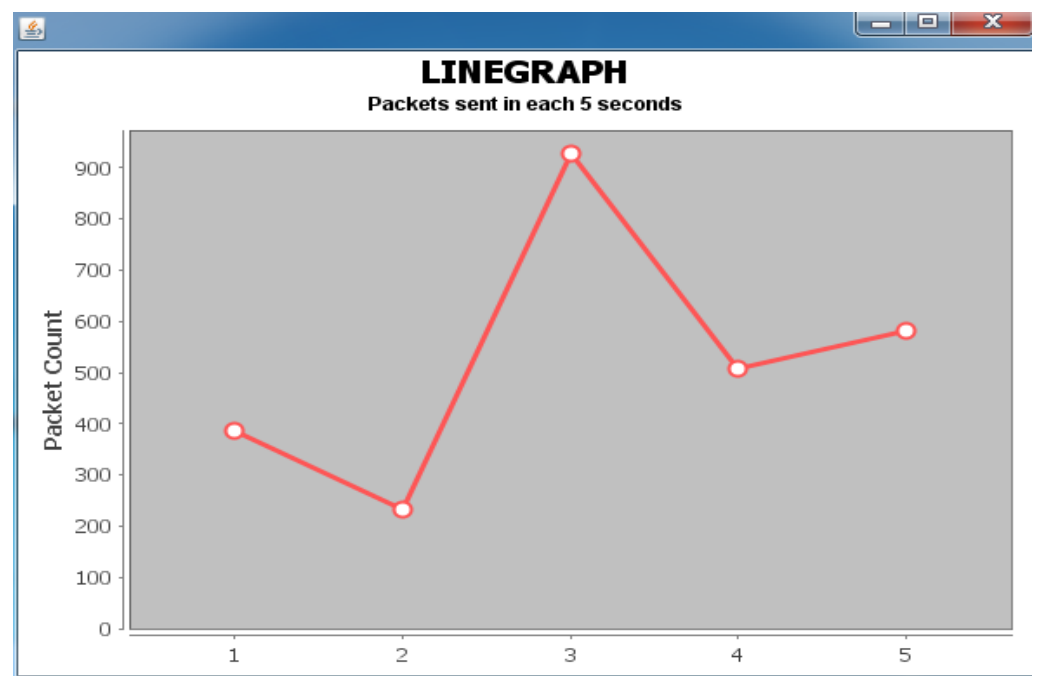

So the data losses occur at higher speed due to which the handover delay occurs in the mobile wimax.

\section{With Using The Save Point}

But when we use the save point between source and the destination then there are no data losses due to which there is no handover delay in the WiMAX. Due to which $100 \%$ of data is received at destination.
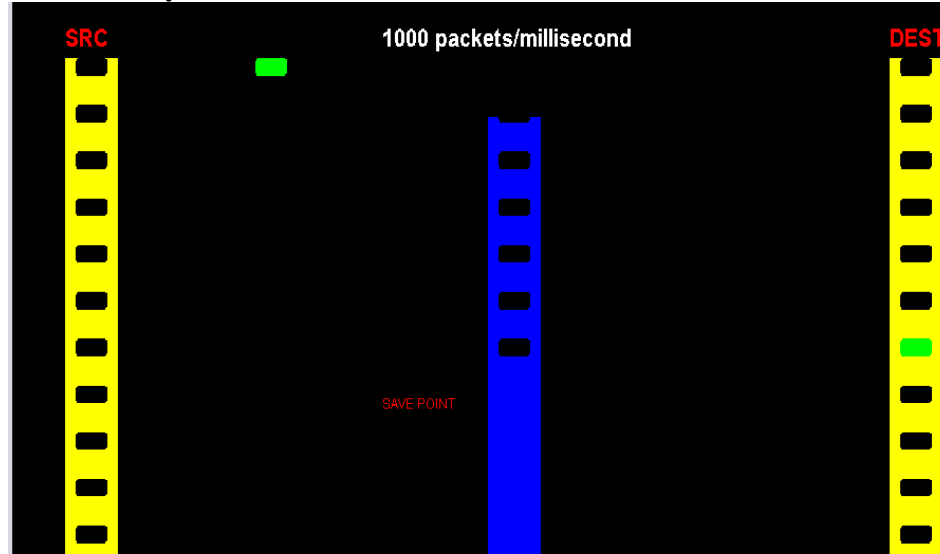

Line graph of the bove diagram is given below which shows that there are no data losses when we use the save point. 


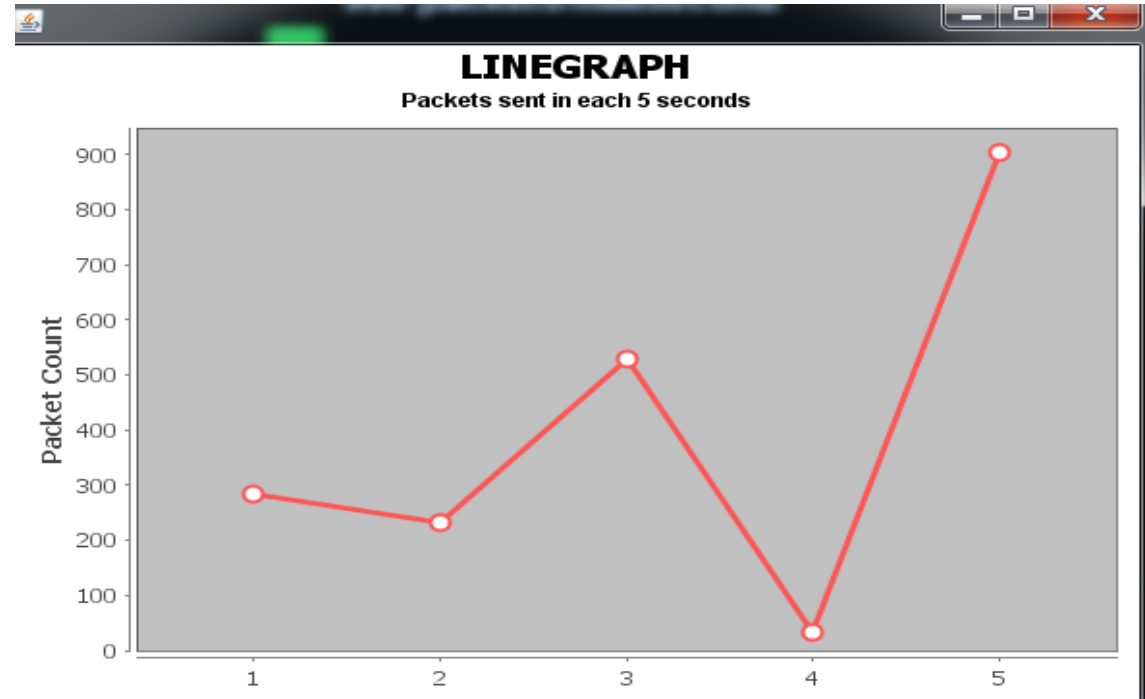

So when we use the save point there would be no data losses hence there would be no handover delay.

\section{Conclusion}

From the above study we have concluded that FEC with retransmission is only successful for light loaded networks but for heavy loaded network we have to use the save point which will provide the better performance and there would be no data losses and no handover delay. In the future we can use the queue to provide the more better performance at more higher speed so that the data losses will negligible.

\section{Refrences}

[1] IEEE 802.16 Working Group, "IEEE Standard for Local and metropolitan Area networks, Part 16: Air Interface for Fixed Broadband Wireless Access Systems,” IEEE Std. 802.16-2004, May 2005.

[2] IEEE 802.16 Working Group, "IEEE Standard for Local and metropolitan Area networks Part 16: Air Interface for Fixed and Mobile Broadband Wireless Access Systems Amendment 2," IEEE Std. 802.16e-2005, Feburary 2006.

[3] G. P. Pollini, "Trends in handover design," Communications Magazine, IEEE, vol. 34, pp. 82-90, 1996.

[4] M. G. Williams, "Directions in media independent handover," IEICE Trans. Fundamentals, vol. E88-A, pp. 1772-1776, July 1, 2005.

[5] D. J. Wright, "Maintaining QoS during handover among multiple wireless access technologies," in Proceedings of Intl. Management of Mobile Business, 2007, pp. 10-10.

[6] R. Hsieh, Z. G. Zhou, and A. Seneviratne, "S-MIP: a seamless handoff architecture for mobile IP," in Proceedings of INFOCOM , 2003, pp. 1774-1784

[7] L. Doo Hwan, K. Kyamakya, and J. P. Umondi, "Fast handover algorithm for IEEE 802.16e broadband wireless access system," in Proceeding of International Symposium on Wireless Pervasive Computing, 2006

[8] S. Minsik, K. Hwasung, and L. Sangho, "A fast handover mechanism for IPv6 based WiBro system," in Proceedings of ICACT,2006.

[9] Zhiwei Yan, Lei Huang, C.-C. Jay Kuo, "Seamless High-Velocity Handover Support in Mobile WiMAX Networks", Xi'an Jiaotong University, Xi'an 710049, P. R. China. 